\title{
Soledad y tecnología en sociedades que envejecen: retos y dificultades de los enfoques socioculturales en la salud pública.
}

\author{
Borja Rivero Jiménez ${ }^{11^{*}}$, \\ David Conde-Caballero ${ }^{22}$, \\ Lorenzo Mariano Juárez ${ }^{3}$ \\ ORCID: http://orcid.org/0000-0002-0837-9372
}

\begin{abstract}
Recibido el 4 noviembre 2020
Aceptado el 7 de diciembre de 2020
\end{abstract}

\section{Resumen}

El envejecimiento de las sociedades contemporáneas es ya un lugar común en la literatura, así como los desafíos que implica para la Salud Pública. Entre ellos, los derivados de la soledad y el aislamiento social se están convirtiendo en un importante tema académico. Las propuestas tecnológicas constituyen una poderosa línea de investigación en el arsenal de soluciones. Una revisión crítica de la literatura sobre la soledad de los ancianos y el papel de las soluciones tecnológicas, discutiendo la necesidad de enfoques socioculturales en los programas de intervención basados en los avances de la investigación cualitativa de una investigación etnográfica en España y Portugal durante los años 2019 y 2020. La literatura ha destacado el papel central que la tecnología tiene que desempeñar en las intervenciones en la esfera de la soledad como problema de salud pública. Sin embargo, la mayoría de los enfoques carecen de perspectivas sociológicas y culturales que pongan en tela de juicio los conceptos de soledad que se abordan, las futuras consecuencias para la salud en los contextos culturales móviles y los retos y cuestiones asociados al uso de la tecnología, como las lagunas que genera en cuestiones de género o étnicas. Las soluciones tecnológicas son una gran promesa para abordar los problemas de salud pública derivados de la soledad, pero es necesario profundizar en los enfoques sociológicos y culturales de cuestiones como el cambio cultural o las limitaciones de la tecnología.

Palabras Clave: Soledad; Tecnología, Cuidado de los mayores; Enfoques socioculturales; Antropología.

${ }^{1}$ Departamento de Ingeniería de Sistemas Informáticos y Telemáticos, Escuela Politécnica, Avda. Universidad s/n, 10003, Universidad de Extremadura, Cáceres.

${ }^{2}$ Departamento de Enfermería, Facultad de Enfermería y Terapia Ocupacional, Avda. Universidad s/n, Universidad de Extremadura, 10003, Cáceres.

${ }^{3}$ Departamento de Enfermería, Facultad de Enfermería y Terapia Ocupacional, Avda. Universidad s/n, Universidad de Extremadura, 10003, Cáceres 


\begin{abstract}
Ageing of contemporary societies is already a commonplace in literature, as well as the challenges that implies for Public Health. Among them, those derived from loneliness and social isolation are becoming an important academic theme. Technological proposals constitute a powerful line of research in the arsenal of solutions. A critical review of the literature on elderly loneliness and the role of technological solutions, discussing the need for sociocultural approaches in intervention programs based on the advances of qualitative research of an ethnographic research in Spain and Portugal during 2019 and 2020. Literature has highlighted the central role that technology has to play in interventions in loneliness as a public health problem. However, most approaches lack sociological and cultural perspectives that call into question the concepts of solitude that are addressed, the future implications for health in movable cultural contexts, and the challenges and issues associated with the use of technology, such as the gaps it generates in gender or ethnic issues. Technological solutions are a great promise for addressing public health problems arising from loneliness, but we need to deepen sociological and cultural approaches to issues such as cultural change or the limitations of technology.
\end{abstract}

Keywords: Loneliness; Technology; Elderly Care; Sociocultural approaches; Anthropology.

\title{
1. Introducción
}

Las sociedades occidentales envejecen a un ritmo acelerado en los últimos años. En España, el Índice de Envejecimiento ha pasado de 37,35 a 120,46 en los últimos 30 años (1978-2018), con un porcentaje de personas mayores sobre la población total de 19,1\% (INE, 2019). Otros países de la cuenca mediterránea presentan cifras muy similares, como son los casos de Italia (22,6\%), Grecia (21,8\%) o Portugal (21,5\%) (Eurostat, 2019). El fenómeno se ha denominado como "segunda transición demográfica" (van de Kaa, 2002), resultado de la confluencia de la disminución de la mortalidad infantil (debido a los avances en condiciones sanitarias, sociales y económicas), una mejora de los estilos de vida y un aumento de la supervivencia en la vejez (Abellán, Ayala y Pujol, 2017). Esta situación se está convirtiendo en un tema central de las políticas públicas y los gobiernos y las organizaciones internacionales han tomado conciencia del desafío que supone en un futuro próximo (Comisión Europea, 2015; Organización Mundial de la Salud, 2015). En países como España, las proyecciones sitúan el porcentaje de personas mayores de 65 años en un 29,4\% para el año 2068 (INE, 2016).

Las consecuencias de este envejecimiento son importantes. Se ha señalado que podríamos estar enfrentando el gran desafío de la salud pública contemporánea (Berrio Valencia, 2012; Bloom, Canning y Lubet, 2015; The Lancet, 2017). La OMS ha incidido reiteradamente en los problemas derivados de vivir en sociedades envejecidas: aumento de la demanda de quienes van a vivir con estados de salud complejos; implicaciones para la prestación de cuidados de calidad; problemas para la sostenibilidad económica de los sistemas de salud; o agudización de determinadas situaciones como la soledad (Organización Mundial de la Salud, 2017), que, no siendo una realidad exclusiva de las personas mayores, es más frecuente entre ellas (Cacioppo et al., 2016; Kharicha et al., 2018; Bandari et al., 2019).

Urgen soluciones para estas problemáticas. La tecnología ha ido tomando cada vez un mayor protagonismo en nuestras vidas. Parece claro que también en las de las personas mayores, que cada vez más conviven con diversa tecnología (Peine, 2020). Se ha desarrollado toda una rama de conocimiento, la Gertontecnología (Kwon, 2017), encargada de estudiar el cuidado de la salud de los mayores a través de dispositivos tecnológicos con el objetivo de que puedan mantenerse el mayor tiempo en sus hogares de manera independiente. Se proponen soluciones para mejorar la calidad de vida de las personas mayores en ámbitos como la detección de emergencias, la monitorización de constantes vitales o la promoción de hábitos saludables. También para uno de los problemas que, en países como Reino Unido (Griffin, 2010) o USA (Putnam, 2000), surge con una importancia creciente en los últimos años: la soledad de las personas mayores.

Se estima que un $6 \%$ del total de la población europea no tiene a quien pedir ayuda o no tiene con quien hablar (Eurostat, 2020). Los gobiernos proponen planes de lucha contra este problema, los tecnólogos diseñan soluciones 
que intentan paliar estos sentimientos de soledad. Nace un espacio en el que los científicos sociales pueden proponer, desde posturas críticas, análisis de las expectativas que estas soluciones tecnológicas están planteando. Este artículo es un intento de dibujar posicionamientos y miradas desde las ciencias sociales para poder aportar algo de luz en las soluciones tecnológicas propuestas para luchar contra la soledad de las personas mayores.

\section{Materiales y métodos}

Hemos realizado una revisión crítica de la bibliografía cualitativa que se ha producido estos últimos años y que trabajaba con enfoques tecnológicos en las propuestas para la solución de la soledad como problema de salud pública en la población envejecida. Para ello, se han realizado búsquedas en las principales bases de datos como SCOPUS, PubMed o Web of Science.

Hemos utilizado datos preliminares de una investigación cualitativa en curso, de carácter etnográfico, en localidades rurales de las regiones de Extremadura (España) y Alentejo (Portugal) durante los años 2019-2020. Los lugares de trabajo sobre el terreno se han elegido sobre la base de datos sociodemográficos, como el porcentaje de la población mayor de 65 años, la densidad de población, el índice de envejecimiento o el índice de juventud. La mayoría de estas poblaciones han sufrido una alta migración, por lo que muchas de las personas mayores que se ha entrevistado tienen redes sociales muy limitadas.

\section{Discusión}

\subsection{Soledad y salud pública}

Entre los problemas señalados está el desafío que la creciente soledad supondrá para la Salud Pública. Algunos medios de comunicación han llegado a utilizar el adjetivo "epidemia” o "enfermedad” cuando hablan de la soledad (Stegmann, 2016; Yanke, 2017). En la literatura podemos encontrar trabajos que se han centrado en la relación entre la soledad y el mayor uso de los recursos de salud (Gerst-Emerson y Jayawardhana, 2015), que han explorado las estrategias y políticas públicas actuales (Patel, Wardle y Parikh, 2019) o que se han enfocado en cómo las industrias y organizaciones pueden contribuir a resolver este problema de Salud Pública (Myers y Palmarini, 2017). Sin embargo, el mayor número de estudios abordan la soledad desde enfoques clínicos, relacionándola con la aparición de diferentes problemas médicos como consecuencia de los cambios que determina en el organismo. Una revisión actual de este objeto de estudio nos muestra como tiene cada vez más espacio dentro del ámbito académico y al que se suman cada vez más disciplinas. Desde el análisis de prensa (Agren, 2017), pasando por la historiografía (Rico Moreno, 2014), el cine (Donio-Bellegarde, Pinazo-Hernandis y Dominguez, 2014), el ocio (Glover, 2018) o las redes sociales (Johansson y Andreasson, 2017), se ha observado la soledad en las sociedades modernas desde enfoques muy diversos.

Algunos estudios han encontrado evidencias de la correspondencia entre la soledad y la aparición de problemas cognitivos (Cacioppo y Hawkley, 2009) o depresión (Dell, Pelham y Murphy, 2019) o cómo la soledad se relaciona con los problemas de deterioro de las capacidades cerebrales (Nilsson et al., 2018). Otra línea de trabajo señala su relación con un posible aumento de la presión sanguínea (Hawkley et al., 2010) o un aumento de las enfermedades coronarias y los accidentes cerebrovasculares (Valtorta et al., 2016). La evidencia de la relación entre la soledad y el aumento del riesgo de mortalidad es cada vez mayor (Leigh-Hunt et al., 2017). Una circunstancia que algunos autores se han atrevido incluso a cuantificar, indicando que la soledad se asocia con un $26 \%$ más de posibilidades de mortalidad temprana, comparable a otros problemas de salud pública como la obesidad o el tabaquismo (HoltLunstad et al., 2015).

Se ha afirmado que la soledad aparece como un verdadero riesgo para la salud pública (Klinenberg, 2016). Existen en este debate dos posturas que se contraponen. De un lado, están quienes defienden la necesidad de que se renueve la agenda de investigación y los estudios se centren más en los riesgos para la salud pública con el objetivo de que el problema sea tomado en serio por los profesionales de salud y los responsables políticos (Valtorta et al., 2016). Por otro lado, se señala cómo la medicalización de la soledad es perjudicial, especialmente en un momento en el que la cuestión está apareciendo en el debate público (McLennan y Ulijaszek, 2018). La medicalización de algunas cuestiones sociales (como, por ejemplo, el VIH/SIDA y la obesidad) no ha funcionado en el pasado para 
convencer de que los problemas de salud pública requieren enfoques integrados y holísticos. Existe en estos momentos oportunidad para poner en el debate público esta preocupación, pero se cree que la medicalización de la soledad desalienta a la colaboración. Es probable que la medicina no tenga ningún efecto como instrumento para abordar por sí sola la ausencia de interacción social.

Coincidimos en este sentido con quienes afirman que existe una noción medicalizada de la soledad, siendo señalada como un síndrome, un problema de salud o un factor de riesgo. Esta noción se relaciona con una homogenización del sentimiento de soledad, atribuible a partir de la objetivación de una emoción tan compleja. Una reflexión sobre el fenómeno de sentirse solo nos señala como es abordado el tema en la literatura existente sobre enfermería y otros campos relacionados con la salud (Karnick, 2005). En la literatura psicológica y de enfermería, la experiencia de la soledad se discute generalmente en términos de relaciones lineales y de causa-efecto e intervenciones prescriptivas.

\section{1. $\quad$ Retos y problemas: expectativas tecnológicas}

La mayoría de los enfoques de las intervenciones se basan en una visión medicalizada y centrada en el individuo. Muestran una tendencia hacia la universalización de los conceptos y la medicalización de un proceso que puede ser prevenido, detectado y tratado (Rodríguez Martín, 2009). Este tipo de enfoque ha sido criticado y se han señalado sus limitaciones, como la debilidad metodológica de las evaluaciones de su eficacia o los problemas derivados de la falta de consenso en la definición de categorías y conceptos (Findlay, 2003; Masi et al., 2013).

Hay grandes expectativas en las posibles soluciones tecnológicas que se pueden dar a la soledad de las personas mayores. Una revisión de la literatura existente nos muestra un número creciente de trabajos que parten de la premisa de que las TIC's son importantes para mantener el contacto social y mejorar el bienestar emocional, compensando de alguna manera el contexto de aislamiento de algunas comunidades rurales (Bornemann, 2014; Berg et al., 2017). En 2007 se desarrolló un experimento en Alemania mediante el uso del correo electrónico, con implicaciones positivas para la socialización y la reducción de los sentimientos de soledad en personas mayores que nunca habían utilizado la tecnología (Fokkema y Knipscheer, 2007). En la última década, las tecnologías desarrolladas para combatir la soledad han sido muy variadas: videojuegos, robots que se comunican con las personas de edad, charlas de apoyo entre pares, teleasistencia, redes sociales específicas o soluciones que informan o recuerdan a la persona las medicinas o las citas médicas pendientes (Chipps, Jarvis y Ramlall, 2017).

Sin embargo, si bien la mayoría de las tecnologías han demostrado cierta eficacia en la reducción del aislamiento social y la soledad, las pruebas actuales no son del todo sólidas. Muchas de las investigaciones apuntan a la necesidad de seguir investigando, ya que sus resultados pueden basarse más en deseos o pequeñas muestras. Los resultados también muestran que la colaboración interdisciplinaria entre áreas es muy escasa, por lo que existe una brecha entre los tecnólogos que desarrollan las tecnologías y otros investigadores que miden el impacto médico, psicológico y psicosocial de estas tecnologías (Khosravi, Rezvani y Wiewiora, 2016).

\subsection{Enfoques socioculturales críticos para las expectativas tecnológicas.}

Nuestra investigación tiene por objeto describir y analizar el contexto de soledad de las personas que envejecen en zonas rurales aisladas de las regiones de Extremadura (España) y Alentejo (Portugal). Con un trabajo interdisciplinar de investigadores en tecnología, ciencias de la salud, antropología y sociología, pretendemos abordar la construcción social de experiencias y categorías vinculadas a la soledad y el impacto que ésta tiene en la salud, trabajando sobre propuestas intervencionistas desde el ámbito tecnológico. A partir de los datos preliminares de la investigación, proponemos una revisión cautelosa de las opiniones excesivamente alarmistas sobre la “epidemia” de la soledad y las opiniones excesivamente optimistas sobre las soluciones tecnológicas.

En cuanto a la primera pregunta, hay que destacar la falta de consenso en la propia definición de la soledad, lo que hace problemático el análisis comparativo de las diferentes realidades. El propio carácter cultural de la experiencia pone en duda las implicaciones futuras para la salud de las poblaciones en la medida en que es una realidad sujeta a cambios culturales (Buz Delgado, 2013) y que, por lo tanto, presenta diferencias según los significados dados por los actores. No se trata, por consiguiente, de una construcción unidimensional, sino de un hecho multidimensional (Rivero Jiménez, Conde Caballero, et al., 2020; Rivero Jiménez, García Perales, et al., 2020). Muchas de las proyecciones futuras se basan en visiones objetivas de la soledad que no dejan espacio para 
las reelaboraciones sociales, lo que tendría, defendemos, una clara relación con los nuevos procesos de morbilidad. En general, debemos superar el reduccionismo de las conceptualizaciones y los problemas metodológicos para medir los efectos en los diferentes contextos culturales (Valtorta et al., 2016). Los enfoques de la salud pública al problema emergente de la soledad deben desarrollarse a partir de una epidemiología social que cuestione las categorías y metodologías a priori de análisis de los efectos sobre la salud. Es necesario comprender la experiencia subjetiva de la soledad y cómo se construye a lo largo de la vida, pero también las peculiaridades de la organización social y cultural de un determinado contexto para conocer sus causas y poder así proponer soluciones que vayan más allá de la intervención individual.

En cuanto al optimismo tecnológico de las propuestas de resolución, defendemos la necesidad de moderar las expectativas y abordar los desafíos actuales. La literatura sobre tecnología y sociedad ha abordado no sólo los problemas de uso social y cultural (Dutton y Reisdorf, 2019; Wilson, Hoffman y Morgenstern, 2019) y la especificidad del contexto de las personas mayores(Barnard et al., 2013; Casamayou, 2017; Martín Martín, 2017), sino también el carácter desintegrador de tales propuestas, que han contribuido a ampliar las brechas sociales, étnicas y culturales (Wilson, Hoffman y Morgenstern, 2019) o de género (Bray, 2007), o incluso las advertencias sobre su carácter colonialista.

\section{Conclusiones}

La soledad es uno de los desafíos de salud pública de presente y de futuro inmediato de las sociedades modernas. En el contexto de poblaciones cada vez más envejecidas e individualizadas, es necesario trabajar sobre los efectos cada vez más evidentes de la soledad en la salud. A partir del trabajo de campo y de la revisión crítica de la literatura hemos argumentado la necesidad de incorporar metodologías y análisis críticos que incorporen las reflexiones teóricas de campos como la sociología y la antropología en temas como la construcción de categorías que permitan el análisis comparativo de estos efectos en contextos interculturales y diacrónicos y en propuestas intervencionistas desde la tecnología, tanto a nivel de efectividad de las intervenciones como de análisis de su impacto social.

\section{Financiación}

Esta investigación se enmarca en el Proyecto "Instituto Internacional de Innovación e Investigación del Envejecimiento (4IE+)” (0499_4IE_PLUS_4_E) financiado por el programa Interreg V-A España-Portugal (POCTEP) 2014-2020.

\section{Referencias}

ABEllán, A., AYAlA, A., PUYOL, R. [online], “Un perfil de las personas mayores en España,2017. Indicadores estadísticos básicos”. 2017. http://envejecimiento.csic.es/documentos/documentos/enredindicadoresbasicos17.pdf [consulta: 2 agosto 2020]

AGREN, A. (2017), «What are we talking about? Constructions of loneliness among older people in the Swedish news-press», Journal of Aging Studies, 41: 18-27.

BANDARI, R., KHANKEH, H.R., SHAHBOULAGHI, F.M., EBADI, A., KESHTKAR, A.A., MONTAZERI, A. (2019), «Defining loneliness in older adults: protocol for a systematic review», Systematic Reviews, 8: 26

BARNARND, Y., BRADLEY, M.D., HODGSON, F., LLOYD, A.D. (2013), «Learning to use new technologies by older adults: Perceived difficulties, experimentation behaviour and usability», Computers in Human Behavior, 29(4): 1715-1724.

BERG, T., WINTERTON, R., PETERSE, M., WARBURTON, J. (2017), «'Although we're isolated, we're not really isolated': The value of information and communication technology for older people in rural Australia», Australasian Journal on Ageing, 36(4): 313-317.

BERRÍO VALENCIA, M. I. (2012) «Aging population: a challenge for public health», Revista Colombiana de Anestesiología, 40(3): 192-194. 
BLOOM, D. E., CANNING, D. LUBET, A. (2015), «Global Population Aging: Facts, Challenges, Solutions \& Perspectives», Daedalus. 144(2): 80-92.

BORNEMANN, R. (2014) The impact of information and communication technology (ICT) usage on social isolation including loneliness in older adults. A systematic review. Berlin, Magdenburg/Stendal University of Applied Sciences.

BRAY, F. (2007) «Gender and Technology», Annual Review of Anthropology, 36:37-53.

BUZ DELGADO, J. (2013), «Envejecimiento y soledad. La importancia de los factores sociales», en Cubillo, M. and Quintanar, F. (eds.), Por una cultura del envejecimiento, México D.F., CMUCH.

CACIOPPO, J T., CACIOPPO, S., ADLER, A. B., LESTER, P. B., MCGURK, D., THOMAS, J. L., CHEN, H. Y. (2016), «The Cultural Context of Loneliness: Risk Factors in Active Duty Soldiers', Journal of Social and Clinical Psychology, 35(10): 865-882.

CACIOPPO, J. T., HAWKLEY, L. C. (2009), «Perceived social isolation and cognition», Trends in Cognitive Sciences, 13(10): 447-454.

CASAMAYOU, A. (2017) «Personas mayores y tecnologías digitales: desafíos de un binomio», Psicología Conocimiento y Sociedad, 7(2):199-226.

CHIPPS, J., JARVIS, M. A. AND RAMLALL, S. (2017), «The effectiveness of e-Interventions on reducing social isolation in older persons: A systematic review of systematic reviews», Journal of Telemedicine and Telecare, 23(10): 817-827.

COMISIÓN EUROPEA (2015), The 2015 Ageing Report Economic and budgetary projections for the 28 EU Member States (2013-2060), Bruselas, Directorate-General for Economic and Financial Affairs of European Comission.

DELL, N. A., PELHAM, M., MURPHY, A. M. (2019), «Loneliness and depressive symptoms in middle aged and older adults experiencing serious mental illness», Psychiatric Rehabilitation Journal, 42(2): 113-120.

DONIO-BELLEGARDE, M., PINAZO-HERNANDIS, S. AND DOMINGUEZ, T. N. (2014), «La representación cinematográfica de la soledad en la vejez: selección de films», Revista INFAD de psicología, 1(2):185-192.

DUTTON, W. H., REISDORF, B. C. (2019), «Cultural divides and digital inequalities: attitudes shaping Internet and social media divides», Information, Communication \& Society, 22(1): 18-38.

EUROSTAT [online] "Population: Structure indicators". 2019. https://ec.europa.eu/eurostat/web/productsdatasets/-/demo_pjanind [consulta:28 septiembre 2019].

EUROSTAT (2020), Ageing Europe. Looking at the lives of older people in the EU, Luxembourg, Publications Office of the European Union.

FINDLAY, R. A. (2003), «Interventions to reduce social isolation amongst older people: Where is the evidence? », Ageing and Society, 23(5): 647-658.

FOKKEMA, T., KNIPSCHEER, K. (2007) 'Escape loneliness by going digital: A quantitative and qualitative evaluation of a Dutch experiment in using ECT to overcome loneliness among older adults', Aging \& Mental Health. Routledge, 11(5), pp. 496-504.

GERST-EMERSON, K., JAYAWARDHANA, J. (2015) «Loneliness as a public health issue: The impact of loneliness on health care utilization among older adults», American Journal of Public Health, 105(5):10131019.

GLOVER, T. D. (2018), «All the Lonely People: Social Isolation and the Promise and Pitfalls of Leisure», Leisure Sciences, 40(1-2): 25-35. doi:

GRIFFIN, J. (2010) The Lonely Society?, London, Mental Health Foundation.

HAWKLEY, L. C., THISTED, R. A., MASI, C. M., CACIOPPO, J. T. (2010), «Loneliness predicts increased blood pressure: 5-year cross-lagged analyses in middle-aged and older adults.», Psychology and Aging, 25(1):132141.

HOLT-LUNSTAD, J., SMITH, T. B., BAKER, M., HARRIS, T., STEPHENSON, D., (2015) «Loneliness and Social Isolation as Risk Factors for Mortality: A Meta-Analytic Review», Perspectives on Psychological Science, 10(2): 227-237. 
INE (2016) [online] “Proyecciones de población 2016-2066”. 2016. http://www.ine.es/prensa/np994.pdf. [consulta: 23 septiembre 2020]

INE (2019) “Estadística del Padrón Continuo”. www.ine.es [consulta: 16 abril 2020].

JOHANSSON, T., ANDREASSON, J. (2017), «The Web of Loneliness: A Netnographic Study of Narratives of Being Alone in an Online Context», Social Sciences, 6(3): 101.

KARNICK, P. M. (2005), «Feeling lonely: Theoretical perspectives», Nursing Science Quarterly, 18(1): 7-12.

KHARICHA, K., MANTHORPE, J., ILIFFE, S., DAVIES, N., WALTERS, K. (2018), «Strategies employed by older people to manage loneliness: systematic review of qualitative studies and model development», International psychogeriatrics, 30(12): 1767-1781.

KHOSRAVI, P., REZVANI, A. WIEWIORA, A. (2016), «The impact of technology on older adults' social isolation», Computers in Human Behavior, 63: 594-603.

KLINENBERG, E. (2016), «Social Isolation, Loneliness, and Living Alone: Identifying the Risks for Public Health», American Journal of Public Health, 106(5): 786-787.

KWON, S. (2017) Gerontechnology: Research, practice, and principles in the field of technology and aging, Springer Publishing Company.

LEIGH-HUNT, N., BAGGULEY, D., BASH, K., TURNER, V., TURNBULL, S., VALTORTA, N. (2017), «An overview of systematic reviews on the public health consequences of social isolation and loneliness», Public Health, 152: 157-171.

MARTÍN MARTÍN, F. M. (2017) «Habilidades comunicativas como condicionantes en el uso de las TIC en personas adultas mayores», International Journal of Educational Research and Innovation, 8:220-232.

MASI, C.M., CHEN, H.Y., HAWKLEY, L.C., CACIOPPO, J.T. (2013), «A Meta Analysis of Interventions to Reduce Loneliness», Personality and social psychology review, 15(3): 1-66.

MCLENNAN, A. K. AND ULIJASZEK, S. J. (2018), «Beware the medicalisation of loneliness», The Lancet, 391(10129): 1480.

MYERS, L., PALMARINI, N. (2017), «Loneliness: Findings from IBM’s Institute for Business Value 2017 Study on Loneliness in the Aging Population», Public Policy \& Aging Report, 27(4): 158-159.

NILSSON, I., LUBORSKY, M., ROSENBERG, L., SANDBERG, L., BOSTRÖM, A.M., BORELL, L. (2018), «Perpetuating harms from isolation among older adults with cognitive impairment: observed discrepancies in homecare service documentation, assessment and approval practices», BMC Health Services Research, 18(1): 800.

ORGANIZACIÓN MUNDIAL DE LA SALUD (2015) Informe sobre el Envejecimiento y la Salud, Ginebra.

ORGANIZACIÓN MUNDIAL DE LA SALUD (2017), Global strategy and action plan on ageing and health, Ginebra.

PATEL, R. S., WARDLE, K., PARIKH, R. J. (2019) «Loneliness: the present and the future», Age and Ageing, 48(4): 476-477.

PEINE, A. (2020), «The co-constitution of ageing and technology - a model and agenda», Ageing \& Society,1-22.

PUTNAM, R. D. (2000), Bowling Alone. The collapse and revival of American Community. New York, Simon \& Schuster Paperbacks.

RICO MORENO, J. (2014), «Hacia una historia de la soledad», Historia y Grafia, 42(21): 35-63.

RIVERO JIMÉNEZ, B., GARCÍA PERALES, N. M., CONDE CABALLERO, D., MUÑOZ GONZÁLEZ, B., CALDERÓN GARCÍA, J. F., MARIANO JUAREZ, L. (2020) «Loneliness Among Rural Elderly: Present and Future Public Health Challenges», en Fonseca, C., Lopes, M.J., Mendes, D., Mendes, F. y García-Alonso, J. (eds.) Handbook of Research on Health Systems and Organizations for an Aging Society. Hershey, IGI Global.

RIVERO JIMÉNEZ, B., CONDE CABALLERO, D., MUÑOZ GONZÁLEZ, B., MARIANO JUÁREZ, L. (2020), «Technology and rural elderly loneliness. thinking about analog solutions for the community», en GarcíaAlonso, J. y Fonseca, C. (eds) Gerontechnology. IWOG 2019. Cham: Springer.

RODRÍGUEZ MARTÍN, M. (2009), «La soledad en el anciano», Gerokomos, 20(4):159-166. 
Stegman, J. G. [online] “La soledad, una enfermedad que podría convertirse en epidemia”. "Periódico ABC”. 2016. https://www.abc.es/sociedad/abci-soledad-enfermedad-podria-convertirse-epidemia201610030235 noticia.html [consulta: 25 septiembre 2020].

THE LANCET (2017) «Ageing: a 21st century public health challenge?», The Lancet. Public health, 2(7): e297.

VALTORTA, N. K., KANAAN, M., GILBODY, S., HANRATTY, B. (2016), «Loneliness, social isolation and social relationships: What are we measuring? A novel framework for classifying and comparing tools», BMJ Open, 6:e010799.

VAN DE KAA, D. J. (2002) «The idea of a second demographic transition in industrialized countries» en Sixth Welfare Policy Seminar of the National Institute of Population and Social Security, Tokyo.

Wilson, B., Hoffman, J., Morgenstern, J. [online] "Predictive Inequity in Object Detection". 2019. http://arxiv.org/abs/1902.11097 [consulta: 28 septiembre 2020).

Yanke, R. [online] "La epidemia de la soledad ya supera a la obesidad como amenaza para la salud". "Periódico El Mundo” https://www.elmundo.es/sociedad/2017/09/21/59c2a0fb46163faa058b45f8.html [consulta: 26 febrero 2020].

\section{Rivero Jiménez, Borja}

brivero@unex.es

Diplomado en Educación Social, Licenciado en Antropología Social y Cultural, Máster Universitario en Investigación, Especialidad Ciencias de la Educación, por la Universidad de Extremadura. Actualmente trabaja en el Instituto Internacional de Investigación e Innovación del Envejecimiento (4IE), un proyecto multidisciplinar que investiga sobre condiciones de salud ligadas al envejecimiento y propone soluciones tecnológicas. Forma parte del Grupo Interdisciplinar de Estudios en Sociedad, Cultura y Salud (GISCSA). Es miembro de la International Commission on Anthropology of Food (ICAF). Formó parte del equipo de investigación del proyecto "Impacto emocional que genera en profesionales de cuidados paliativos el contacto diario con el sufrimiento y con la muerte: Análisis de estrategias de autocuidado". Ha desarrollado investigación y publicado desde enfoques cualitativos sobre salud y envejecimiento, soledad, alimentación o cuidado. Ha sido galardonado con el premio Internacional de investigación aplicada de la Asociación Portuguesa de Antropología 2020

\section{Conde Caballero, David}

dcondecab@unex.es

Enfermero Podólogo, Máster en Antropología de la Salud, Enfermero especialista en Riesgos Laborales, Doctor en Antropología por la Universidad Nacional de Educación a Distancia (España) con una Tesis Doctoral calificada como Sobresaliente Cum Laude. Es autor de un buen número de artículos científicos, capítulos de libros y libros completos. Ha presentado múltiples comunicaciones en diferentes congresos internacionales y reuniones científicas. Premio de investigación internacional “Arturo Barea 2019”. Premio Internacional de investigación aplicada de la Asociación Portuguesa de Antropología 2020. Con más de 15 años de experiencia clínica, en la actualidad en Profesor Contratado Doctor en la Universidad de Extremadura. Sus intereses de investigación se mueven en el ámbito de la alimentación, los cuidados en el envejecimiento y los riesgos laborales en Enfermería. Ha participado en diversos proyectos de investigación regionales, nacionales e internacionales. Es miembro en la actualidad de la International Commission on Anthropology of Food (ICAF), la International Network on Hunger and Cultural Studies (CIEMEDH-UNED), del Grupo de Investigación en Sociedad, Cultura y Salud (GISCA) y del Instituto Internacional de Investigación e Innovación del Envejecimiento (4iE+).

\section{Mariano Juárez, Lorenzo}

\section{lorenmariano@unex.es}

Diplomado en Enfermería, Doctor en Antropología. En la actualidad es Coordinador de Relaciones con Iberoamérica de la International Commision of Anthropology of Food and Nutrition (ICAF) y Secretario Académico del Departamento de Enfermería. Ha sido Subdirector de la Oficina de Cooperación al Desarrollo y el Voluntariado de 
la Uex durante dos años. Es autor de más de 90 publicaciones que incluyen artículos científicos, libros y capítulos de libro y ha participado como revisor de revistas de ciencias sociales y humanidades. Ha sido Editor Coordinador de la Revista de Ciencias Sociales Revibe. Premio Extraordinario de doctorado, fue el ganador de la segunda edición de Premio a la mejor Tesis Doctoral del Grupo G9 de Universidades. Ha sido Profesor visitante en la Universidad San Carlos de Guatemala y Visiting Scholar en Center for Human Rights and Peace Studies, en CUNY, New York. Miembro del grupo de investigación "Etnología Americana", forma parte del "Observatorio de la Alimentación en México” y del “Comité Científico de Acción contra el Hambre” en Guatemala, Trabaja desde 2004 en la región Maya Ch’orti’ del oriente de Guatemala, interesado en cuestiones como el hambre, la antropología de la alimentación y del desarrollo o las lógicas y sentidos de la violencia, ámbitos que constituyen sus líneas de investigación americanista. En España, realiza investigación en el ámbito de la salud mental, narrative medicine, la gerontecnología o la salud intercultural. Es el coordinador del grupo de Investigación GIGCSA. 